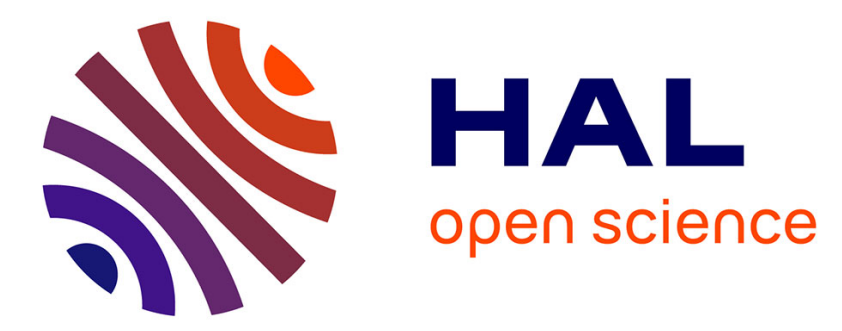

\title{
RFC7631: TLV Naming in the Mobile Ad Hoc Network (MANET) Generalized Packet/Message Format
}

Christopher Chris Dearlove, Thomas Heide Clausen

\section{To cite this version:}

Christopher Chris Dearlove, Thomas Heide Clausen. RFC7631: TLV Naming in the Mobile Ad Hoc Network (MANET) Generalized Packet/Message Format. [Technical Report] RFC7631, The Internet Engineering Task Force. 2015. hal-03172475

HAL Id: hal-03172475

https://hal-polytechnique.archives-ouvertes.fr/hal-03172475

Submitted on 17 Mar 2021

HAL is a multi-disciplinary open access archive for the deposit and dissemination of scientific research documents, whether they are published or not. The documents may come from teaching and research institutions in France or abroad, or from public or private research centers.
L'archive ouverte pluridisciplinaire HAL, est destinée au dépôt et à la diffusion de documents scientifiques de niveau recherche, publiés ou non, émanant des établissements d'enseignement et de recherche français ou étrangers, des laboratoires publics ou privés. 
Internet Engineering Task Force (IETF)

C. Dearlove

Request for Comments: 7631

BAE Systems ATC

Updates: 5444

Category: Standards Track

T. Clausen

ISSN: $2070-1721$

LIX, Ecole Polytechnique

September 2015

TLV Naming in the Mobile Ad Hoc Network (MANET)

Generalized Packet/Message Format

Abstract

This document reorganizes the naming of already-allocated TLV (typelength-value) types and type extensions in the "Mobile Ad hoc NETwork (MANET) Parameters" registries defined by RFC 5444 to use names appropriately. It has no consequences in terms of any protocol implementation.

This document also updates the Expert Review guidelines in RFC 5444, so as to establish a policy for consistent naming of future TLV type and type extension allocations. It makes no other changes to RFC 5444 .

Status of This Memo

This is an Internet Standards Track document.

This document is a product of the Internet Engineering Task Force (IETF). It represents the consensus of the IETF community. It has received public review and has been approved for publication by the Internet Engineering Steering Group (IESG). Further information on Internet Standards is available in Section 2 of RFC 5741.

Information about the current status of this document, any errata, and how to provide feedback on it may be obtained at http://www.rfc-editor.org/info/rfc7631. 
Copyright Notice

Copyright (c) 2015 IETF Trust and the persons identified as the document authors. All rights reserved.

This document is subject to BCP 78 and the IETF Trust's Legal Provisions Relating to IETF Documents

(http://trustee.ietf.org/license-info) in effect on the date of publication of this document. Please review these documents carefully, as they describe your rights and restrictions with respect to this document. Code Components extracted from this document must include Simplified BSD License text as described in section $4 . e$ of the Trust Legal Provisions and are provided without warranty as described in the Simplified BSD License.

Table of Contents

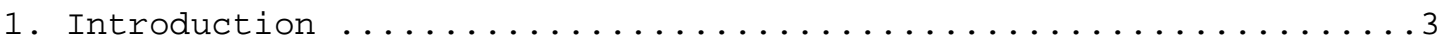

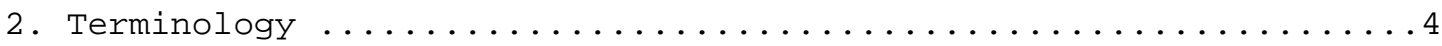

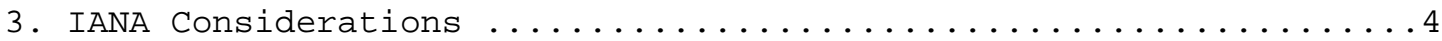

3.1. Expert Review: Evaluation Guidelines ................

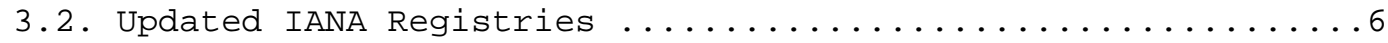

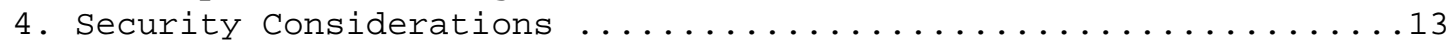

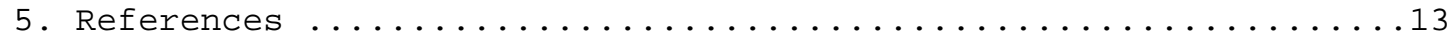

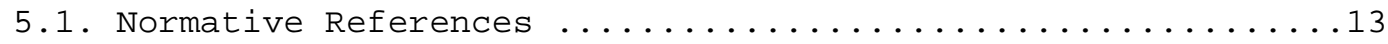

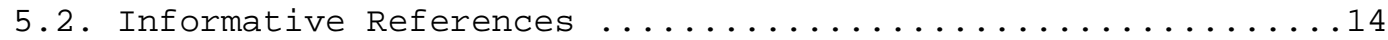

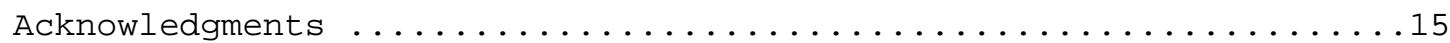

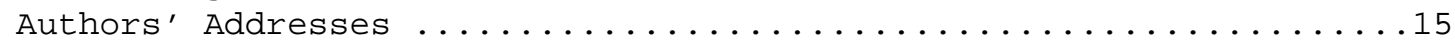




\section{Introduction}

This document reorganizes and rationalizes the naming of TLVs (typelength-value structures) defined by [RFC5444] and recorded by IANA in the following subregistries of the "Mobile Ad hoc NETwork (MANET) Parameters" registry: "Packet TLV Types", "Message TLV Types", and "Address Block TLV Types".

This document reorganizes the naming of already-allocated Packet, Message, and Address Block TLV types, and their corresponding type extensions. It also updates the corresponding IANA registries.

TLVs have a type (one octet) and a type extension (one octet) that together form a full type (of two octets). A TLV may omit the type extension when it is zero. However, that applies only to its representation; it still has a type extension of zero. A TLV type defines an IANA registry of type extensions for that type.

There have been two forms of TLV allocation.

The first, but less common, form of allocation has been that allocation of the TLV type has defined (but not necessarily allocated) all the type extensions for that TLV type. This applies, for example, to the Address Block TLV LINK_METRIC specified in [RFC7181]. The LINK_METRIC type extensions are all available for allocation for different definitions of link metric. It is appropriate in this case to apply the name LINK_METRIC to the type, and also to all the full types corresponding to that type, as has been done. Type extensions can then be individually named or can be simply referred to by their number.

The second, more common, form of allocation has been that allocation of the TLV type has defined only type extension 0, and possibly type extension 1, for that TLV type. An example is the Address Block TLV LINK_STATUS defined in [RFC6130], where only type extension 0 is allocated. It is not reasonable to assume that the remaining 255 type extensions will be allocated to forms of LINK_STATUS. (Other forms of link status are already catered to by the introduction, in [RFC7188], of a registry for values of the LINK_STATUS TLV.) Thus, the name LINK_STATUS should be attached to the specific type extension for that type, i.e., to the full type and not to the TLV type when used with any other type extensions. This was, however, not done as part of the initial registration of this TLV type. Effectively, this leaves, for the LINK_STATUS TLV type, the type extensions 1-255 either unavailable for allocation (if applying strictly the interpretation that they must relate to a LINK_STATUS) or counterintuitively named for their intended function. 
The purpose of this document is to change how names of the second form are applied and recorded in IANA registries, and to provide guidelines and instructions for future TLV type allocations. This is to facilitate the addition of new TLVs using type extensions other than 0, but without them having inappropriate names attached. So, for example, LINK_STATUS will become the name of the full type (composed of the TLV type 3 and the TLV type extension 0 ) and will cease being the name of the TLV type 3. This leaves the question of how to name the type. As it is not clear what other TLVs might be defined for other type extensions of the same type, the type is currently left unnamed and specified only by number.

This document also updates the Expert Review guidelines from [RFC544], so as to establish a policy for consistent naming of future TLV type and type extension allocations.

For clarity, all currently allocated TLVs in [RFC5497], [RFC6130], [RFC6621], [RFC7181], and [RFC7182] are listed in the IANA Considerations section of this document, each specifying the updates or indicating no change when that is appropriate (such as the LINK_METRIC TLV and both TLVs defined in [RFC6621]). The only changes are of naming.

Note that nothing in this document changes the operation of any protocol. This naming is already used, in effect, in [RFC6130] and [RFC7181], currently the main users of allocated TLVs. For example, the former indicates that all usage of LINK_STATUS refers to that TLV with type extension 0 .

\section{Terminology}

The key words "MUST", "MUST NOT", "REQUIRED", "SHALL", "SHALL NOT", "SHOULD", "SHOULD NOT", "RECOMMENDED", "NOT RECOMMENDED", "MAY", and "OPTIONAL" in this document are to be interpreted as described in [RFC2119].

All references to elements such as "packet", "message", and "TLV" in this document refer to those defined in [RFC5444].

3. IANA Considerations

This document updates the Expert Review evaluation guidelines for allocations in [RFC5444] in the "Packet TLV Types", "Message TLV Types", and "Address Block TLV Types" registries and updates the already-made allocations to conform with these guidelines. 
3.1. Expert Review: Evaluation Guidelines

For registration in the "Packet TLV Types", "Message TLV Types", and "Address Block TLV Types" registries, the following guidelines apply, in addition to those given in Section 6.1 in [RFC5444]:

- If the requested TLV type immediately defines (but not necessarily allocates) all the corresponding type extensions for versions of that type, then a common name SHOULD be assigned for the TLV type.

This case is unchanged by this specification. This currently includes TLV types named ICV, TIMESTAMP, and LINK_METRIC; it also includes the HELLO Message-Type-specific TLVs defined in [RFC6621].

- Otherwise, if the requested TLV type does not immediately define all the corresponding type extensions for versions of that type, then a common name SHOULD NOT be assigned for that TLV type. Instead, it is RECOMMENDED that:

* The "description" for the allocated TLV type be "Defined by Type Extension".

* For Packet TLV Types, the type extension registry, created for the TLV type, be named "Type XX Packet TLV Type Extensions", with $\mathrm{XX}$ replaced by the numerical value of the TLV type.

* For Message TLV Types, the type extension registry, created for the TLV type, be named "Type XX Message TLV Type Extensions", with XX replaced by the numerical value of the TLV type.

* For Address Block TLV Types, the type extension registry, created for the TLV type, be named "Type XX Address Block TLV Type Extensions", with XX replaced by the numerical value of the TLV type.

* When a new type extension is required, unless there are reasons to the contrary, the next consecutive type extension is allocated and given a name. (Reasons to the contrary MAY include maintaining a correspondence between corresponding Packet, Message, and Address Block TLVs, and reserving type extension zero if not yet allocated.) 


\subsection{Updated IANA Registries}

The following changes (including correction of some existing minor errors) apply to the IANA registry "Mobile Ad hoc NETwork (MANET) Parameters". For clarity, registries that are unchanged, including those that define all type extensions of a TLV type, are listed as unchanged.

The IANA registry "Packet TLV Types" is unchanged.

The IANA registry "ICV Packet TLV Type Extensions" is unchanged.

The IANA registry "TIMESTAMP Packet TLV Type Extensions" is unchanged.

The IANA registry "Message TLV Types" is changed to match Table 1.

\begin{tabular}{|c|l|c|} 
Type & Description & Reference \\
0 & Defined by Type Extension & [RFC5497] \\
1 & Defined by Type Extension & [RFC5497] \\
$2-4$ & Unassigned & [RFC7182] \\
5 & ICV & [RFC7182] \\
7 & TIMESTAMP & [RFC7181] \\
8 & Defined by Type Extension & [RFC7181] \\
$9-223$ & Unassigned & [RFC5444] \\
$224-255$ & Reserved for Experimental Use &
\end{tabular}

Table 1: Message TLV Types 
The IANA registry "INTERVAL_TIME Message Type Extensions" has been renamed "Type 0 Message TLV Type Extensions" and changed to match Table 2 .

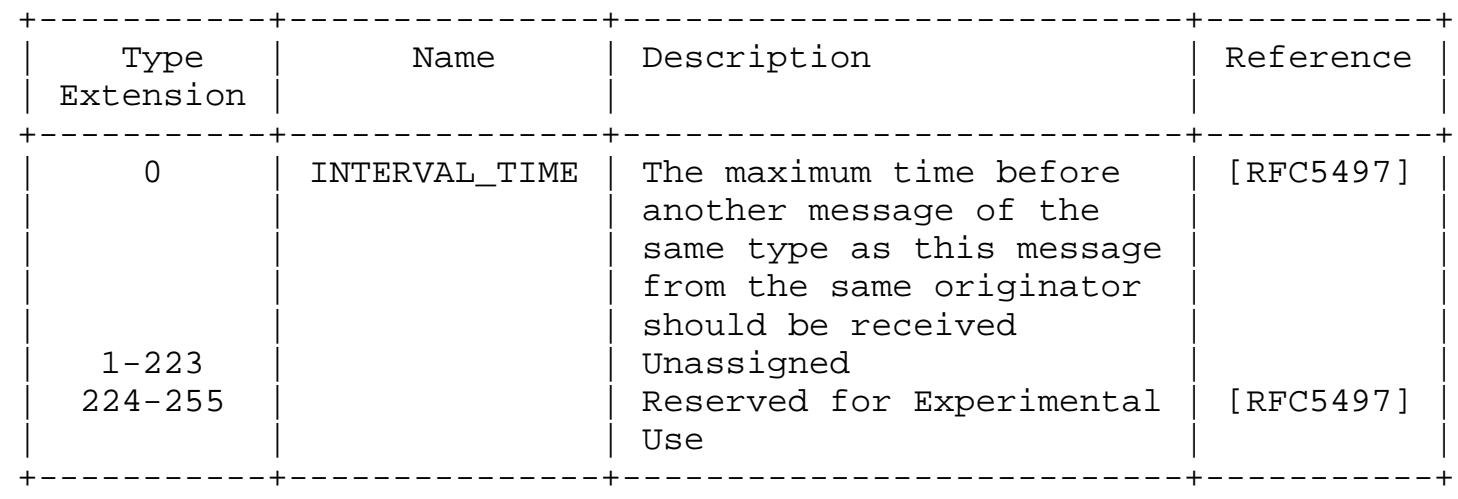

Table 2: Type 0 Message TLV Type Extensions

The IANA registry "VALIDITY_TIME Message Type Extensions" has been renamed "Type 1 Message TLV Type Extensions" and changed to match Table 3 .

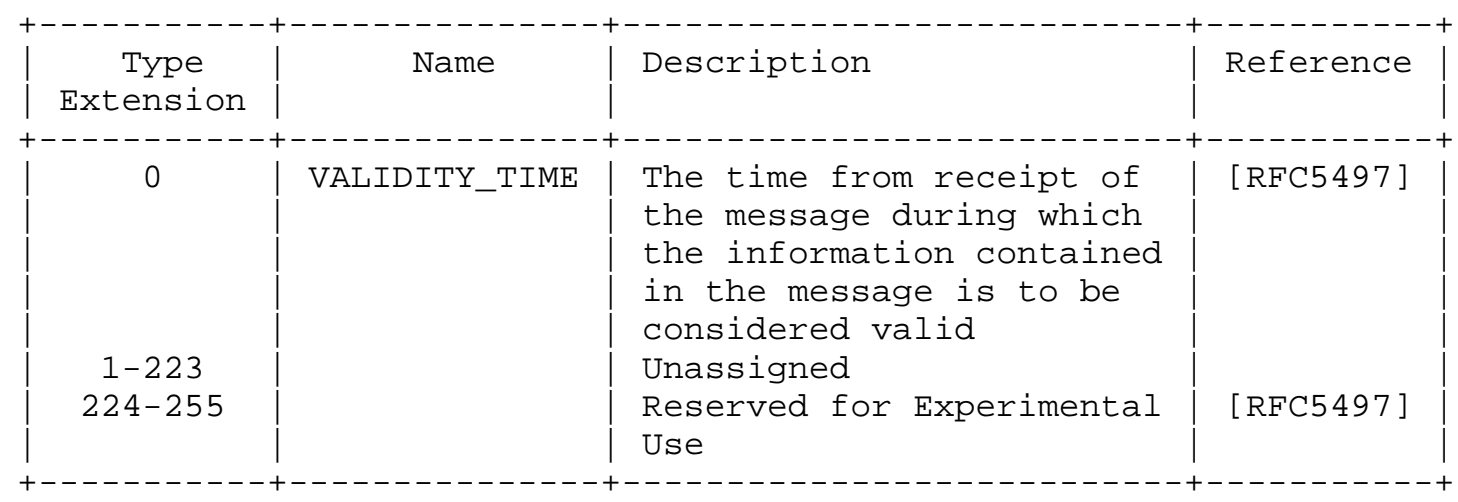

Table 3: Type 1 Message TLV Type Extensions

The IANA registry "ICV Message TLV Type Extensions" is unchanged.

The IANA registry "TIMESTAMP Message TLV Type Extensions" is unchanged. 
The IANA registry "MPR_WILLING Message Type Extensions" has been renamed "Type 7 Message TLV Type Extensions" and changed to match Table 4 .

\begin{tabular}{|c|c|c|c|}
\hline $\begin{array}{c}\text { Type } \\
\text { Extension }\end{array}$ & Name & Description & Reference \\
\hline $1-223$ & MPR_WILLING & $\begin{array}{l}\text { Bits } 0-3 \text { specify the } \\
\text { originating router's } \\
\text { willingness to act as a } \\
\text { flooding MPR; bits } 4-7 \\
\text { specify the originating } \\
\text { router's willingness to act } \\
\text { as a routing MPR } \\
\text { Unassigned }\end{array}$ & [RFC7181] \\
\hline $224-255$ & & $\begin{array}{l}\text { Reserved for Experimental } \\
\text { Use }\end{array}$ & [RFC7181] \\
\hline
\end{tabular}

Table 4: Type 7 Message TLV Type Extensions

The IANA registry "CONT_SEQ_NUM Message Type Extensions" has been renamed "Type 8 Message TLV Type Extensions" and changed to match Table 5 .

\begin{tabular}{|c|c|c|c|}
\hline $\begin{array}{c}\text { Type } \\
\text { Extension }\end{array}$ & Name & Description & Reference \\
\hline 0 & $\begin{array}{l}\text { CONT_SEQ_NUM } \\
\text { (COMPLETE) }\end{array}$ & $\begin{array}{l}\text { Specifies a content } \\
\text { sequence number for this } \\
\text { complete message }\end{array}$ & [RFC7181] \\
\hline $\begin{array}{c}1 \\
2-223\end{array}$ & $\begin{array}{l}\text { CONT_SEQ_NUM } \\
\text { (INCOMPLETE) }\end{array}$ & $\begin{array}{l}\text { Specifies a content } \\
\text { sequence number for this } \\
\text { incomplete message } \\
\text { Unassigned }\end{array}$ & [RFC7181] \\
\hline $224-255$ & & $\begin{array}{l}\text { Reserved for Experimental } \\
\text { Use }\end{array}$ & [RFC7181] \\
\hline
\end{tabular}

Table 5: Type 8 Message TLV Type Extensions

The IANA registry "HELLO Message-Type-specific Message TLV Types" is unchanged.

The IANA registry "SMF_TYPE Message TLV Type Extensions" is unchanged. 
The IANA registry "TC Message-Type-specific Message TLV Types" is unchanged.

The IANA registry "Address Block TLV Types" has been changed to match Table 6 .

\begin{tabular}{|c|c|c|}
\hline Type & Description & Reference \\
\hline 0 & Defined by Type Extension & {$[\mathrm{RFC5} 497]$} \\
\hline 1 & Defined by Type Extension & {$[\mathrm{RFC} 5497]$} \\
\hline 2 & Defined by Type Extension & {$[\mathrm{RFC} 6130]$} \\
\hline 3 & Defined by Type Extension & {$[\mathrm{RFC} 6130]$} \\
\hline 4 & Defined by Type Extension & {$[\mathrm{RFC} 6130]$} \\
\hline 5 & $\mathrm{ICV}$ & {$[\mathrm{RFC} 7182]$} \\
\hline 6 & TIMESTAMP & {$[\mathrm{RFC} 7182]$} \\
\hline 7 & LINK_METRIC & {$[\mathrm{RFC} 7181]$} \\
\hline 8 & Defined by Type Extension & {$[\mathrm{RFC} 7181]$} \\
\hline 9 & Defined by Type Extension & {$[\mathrm{RFC} 7181]$} \\
\hline 10 & Defined by Type Extension & {$[\mathrm{RFC} 7181]$} \\
\hline $11-223$ & Unassigned & \\
\hline $224-255$ & Reserved for Experimental Use & [RFC5444] \\
\hline
\end{tabular}

Table 6: Address Block TLV Types

The IANA registry "INTERVAL_TIME Address Block TLV Type Extensions" has been renamed "Type 0 Address Block TLV Type Extensions" and changed to match Table 7 .

\begin{tabular}{|c|c|c|c|}
\hline $\begin{array}{c}\text { Type } \\
\text { Extension }\end{array}$ & Name & Description & Reference \\
\hline $1-223$ & INTERVAL_TIME & $\begin{array}{l}\text { The maximum time before } \\
\text { another message of the } \\
\text { same type as this message } \\
\text { from the same originator } \\
\text { and containing this } \\
\text { address should be } \\
\text { received } \\
\text { Unassigned }\end{array}$ & [RFC5497] \\
\hline $224-255$ & & $\begin{array}{l}\text { Reserved for Experimental } \\
\text { Use }\end{array}$ & [RFC5497] \\
\hline
\end{tabular}

Table 7: Type 0 Address Block TLV Type Extensions 
The IANA registry "VALIDITY_TIME Address Block TLV Type Extensions" has been renamed "Type 1 Address Block TLV Type Extensions" and changed to match Table 8 .

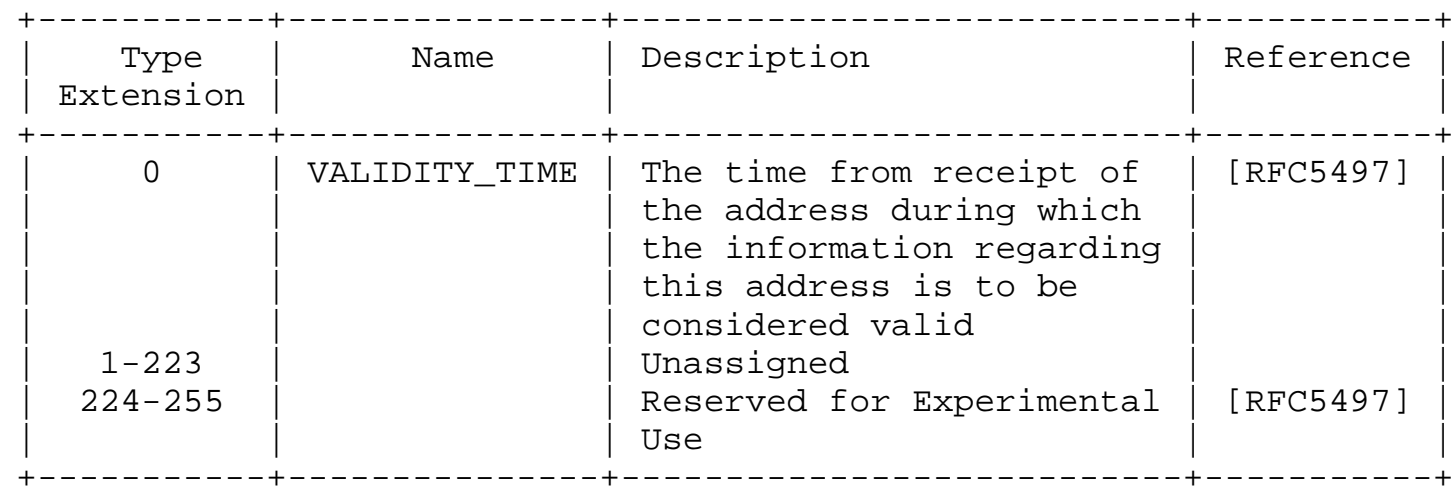

Table 8: Type 1 Address Block TLV Type Extensions

The IANA registry "LOCAL_IF Address Block TLV Type Extensions" has been renamed "Type 2 Address Block TLV Type Extensions" and changed to match Table 9 .

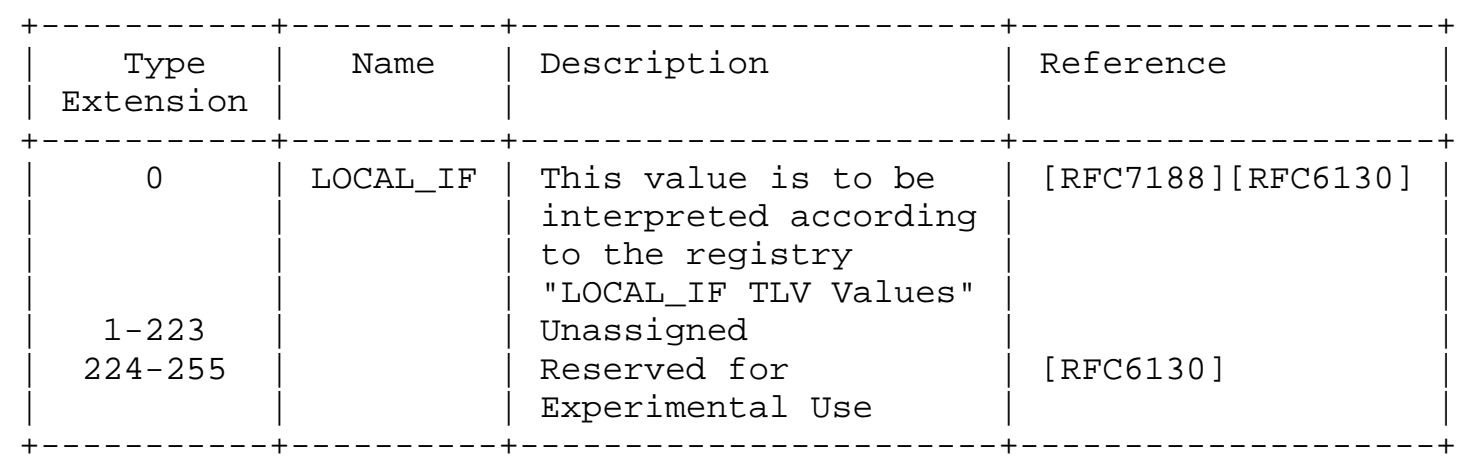

Table 9: Type 2 Address Block TLV Type Extensions 
The IANA registry "LINK_STATUS Address Block TLV Type Extensions" has been renamed "Type 3 Address Block TLV Type Extensions" and changed to match Table 10 .

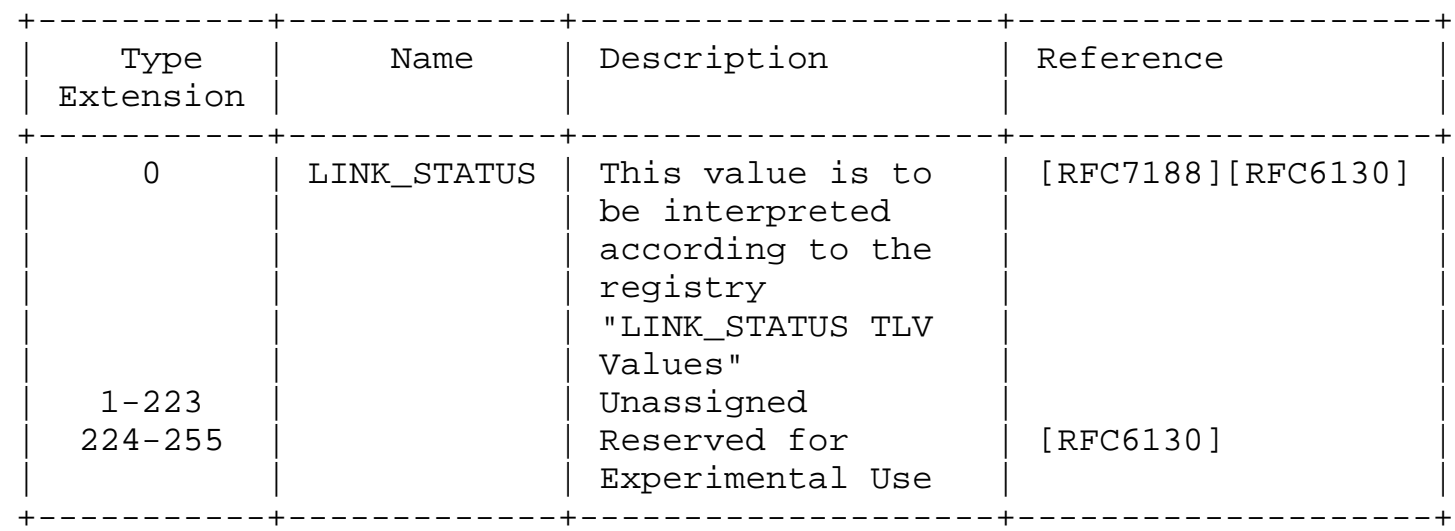

Table 10: Type 3 Address Block TLV Type Extensions

The IANA registry "OTHER_NEIGHB Address Block TLV Type Extensions" has been renamed "Type 4 Address Block TLV Type Extensions" and changed to match Table 11 .

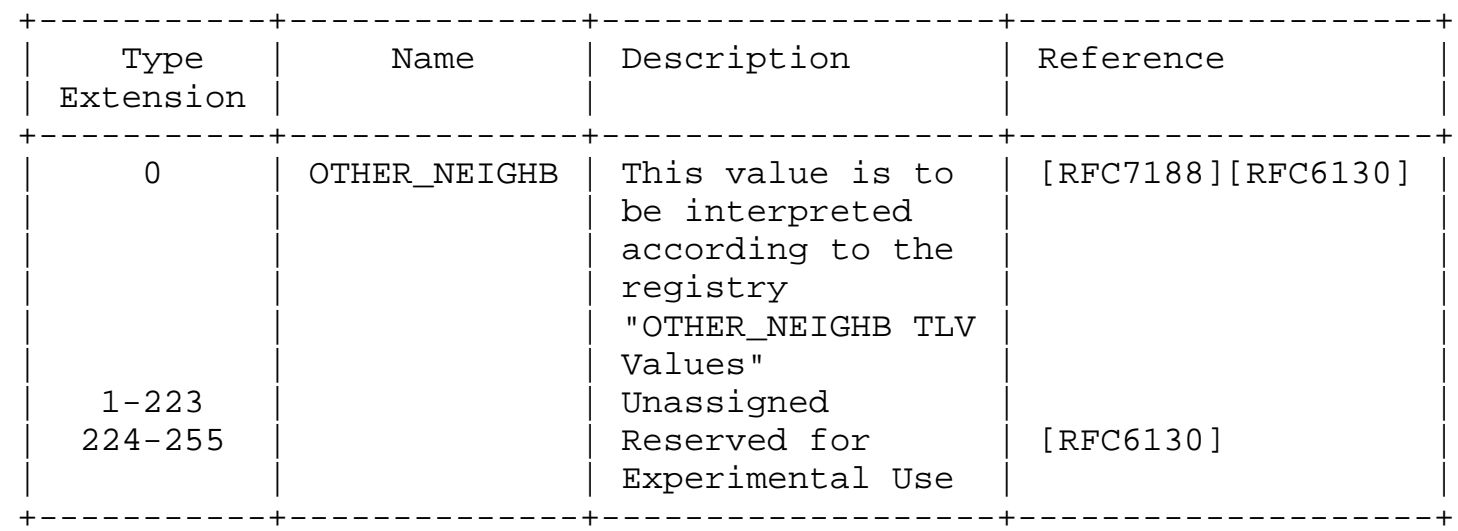

Table 11: Type 4 Address Block TLV Type Extensions

The IANA registry "ICV Address TLV Type Extensions" has been renamed "ICV Address Block TLV Type Extensions" but is otherwise unchanged.

The IANA registry "TIMESTAMP Address TLV Type Extensions" has been renamed "TIMESTAMP Address Block TLV Type Extensions" but is otherwise unchanged. 
The IANA registry "LINK_METRIC Address Block TLV Type Extensions" is unchanged.

The IANA registry "MPR Address Block TLV Type Extensions" has been renamed "Type 8 Address Block TLV Type Extensions" and changed to match Table 12 .

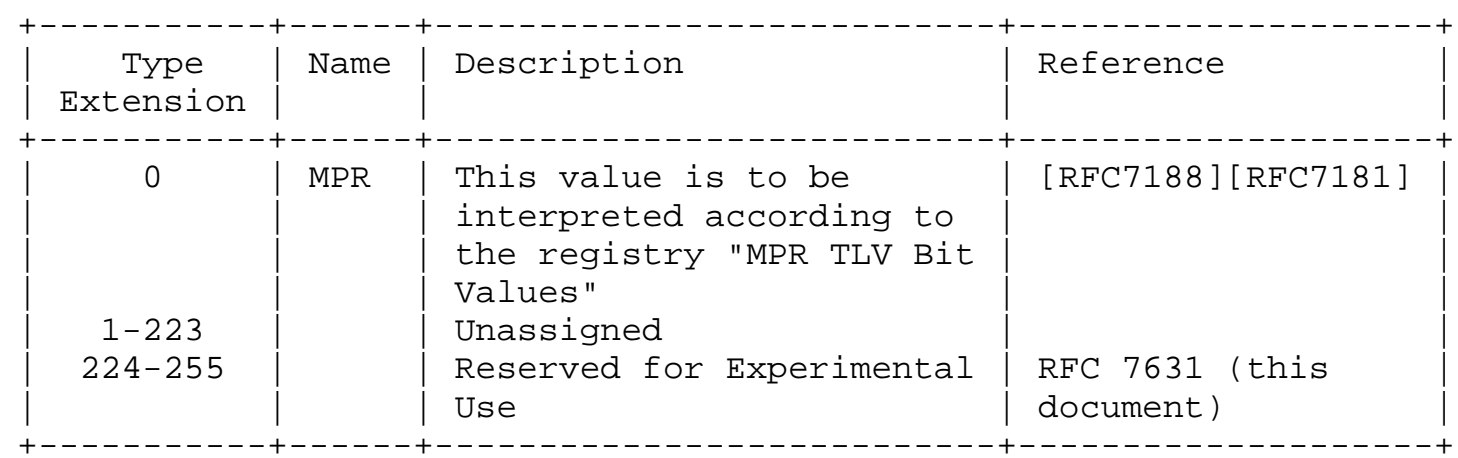

Table 12: Type 8 Address Block TLV Type Extensions

The IANA registry "NBR_ADDR_TYPE Address Block TLV Type Extensions" has been renamed "Type 9 Address Block TLV Type Extensions" and changed to match Table 13 .

\begin{tabular}{|c|c|c|c|}
\hline $\begin{array}{c}\text { Type } \\
\text { Extension }\end{array}$ & Name & Description & Reference \\
\hline $\begin{array}{c}1-223 \\
224-255\end{array}$ & NBR_ADDR_TYPE & $\begin{array}{l}\text { This value is to } \\
\text { be interpreted } \\
\text { according to the } \\
\text { registry } \\
\text { "NBR_ADDR_TYPE } \\
\text { Address Block } \\
\text { TLV Bit Values" } \\
\text { Unassigned } \\
\text { Reserved for } \\
\text { Experimental Use }\end{array}$ & $\begin{array}{l}\text { RFC } 7631 \text { (this } \\
\text { document) }\end{array}$ \\
\hline
\end{tabular}

Table 13: Type 9 Address Block TLV Type Extensions 
The IANA registry "GATEWAY Address Block TLV Type Extensions" has been renamed "Type 10 Address Block TLV Type Extensions" and changed to match Table 14 .

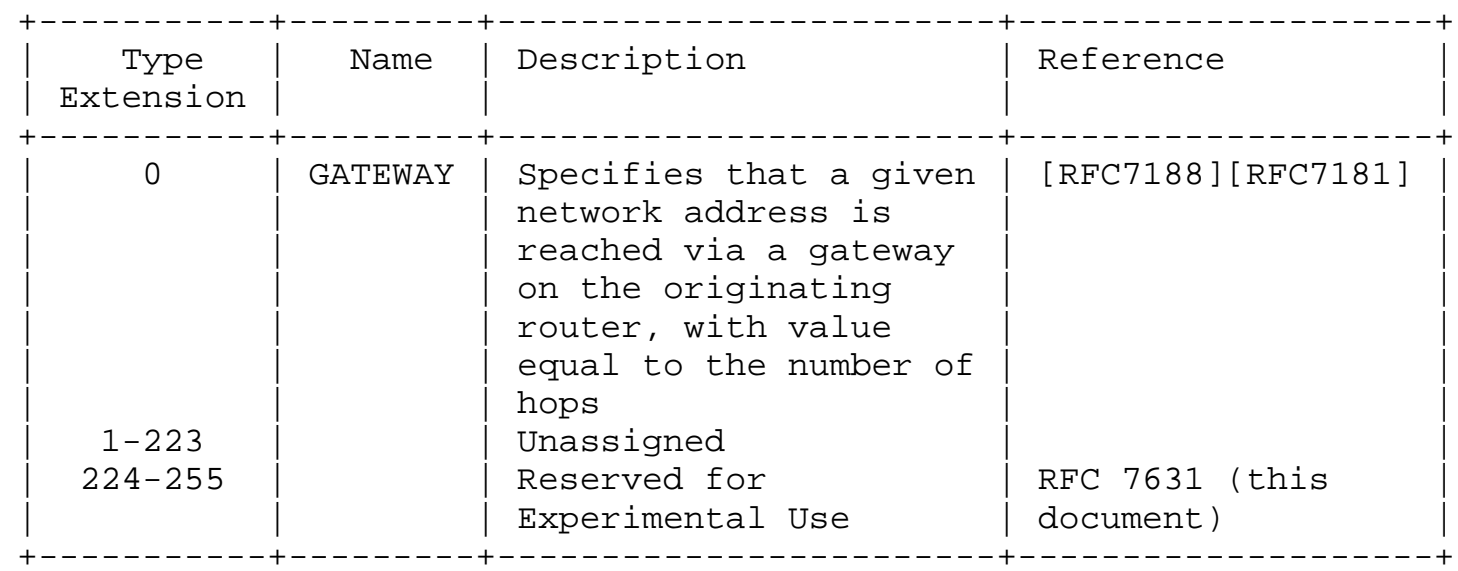

Table 14: Type 10 Address Block TLV Type Extensions

The IANA registry "HELLO Message-Type-specific Address Block TLV Types" is unchanged.

The IANA registry "SMF_NBR_TYPE Address Block TLV Type Extensions" is unchanged.

The IANA registry "TC Message-Type-specific Address Block TLV Types" is unchanged.

Note: This document adds reservations for Experimental Use [RFC5226], omitted in [RFC7181], to the last three tables.

4. Security Considerations

As this document is concerned only with how entities are named, those names being used only in documents such as this and IANA registries, this document has no security considerations.

5. References

5.1. Normative References

[RFC2119] Bradner, S., "Key words for use in RFCs to Indicate Requirement Levels", BCP 14, RFC 2119, DOI 10.17487/RFC2119, March 1997, <http://www.rfc-editor.org/info/rfc2119>. 
[RFC5444] Clausen, T., Dearlove, C., Dean, J., and C. Adjih, "Generalized Mobile Ad Hoc Network (MANET) Packet/Message Format", RFC 5444, DOI 10.17487/RFC5444, February 2009, <http: //wWw.rfc-editor.org/info/rfc5444>.

[RFC5497] Clausen, T. and C. Dearlove, "Representing Multi-Value Time in Mobile Ad Hoc Networks (MANETs)", RFC 5497, DOI 10.17487/RFC5497, March 2009, <http: //www.rfc-editor.org/info/rfc5497>.

[RFC6130] Clausen, T., Dearlove, C., and J. Dean, "Mobile Ad Hoc Network (MANET) Neighborhood Discovery Protocol (NHDP)", RFC 6130, DOI 10.17487/RFC6130, April 2011, <http://www.rfc-editor.org/info/rfc6130>.

[RFC6621] Macker, J., Ed., "Simplified Multicast Forwarding", RFC 6621, DOI 10.17487/RFC6621, May 2012, <http://www.rfc-editor.org/info/rfc6621>.

[RFC7181] Clausen, T., Dearlove, C., Jacquet, P., and U. Herberg, "The Optimized Link State Routing Protocol Version 2", RFC 7181, DOI 10.17487/RFC7181, April 2014, <http://www.rfc-editor.org/info/rfc7181>.

[RFC7182] Herberg, U., Clausen, T., and C. Dearlove, "Integrity Check Value and Timestamp TLV Definitions for Mobile Ad Hoc Networks (MANETs)", RFC 7182, DOI 10.17487/RFC7182, April 2014, <http://www.rfc-editor.org/info/rfc7182>.

[RFC7188] Dearlove, C. and T. Clausen, "Optimized Link State Routing Protocol Version 2 (OLSRv2) and MANET Neighborhood Discovery Protocol (NHDP) Extension TLVs", RFC 7188, DOI 10.17487/RFC7188, April 2014, <http://www.rfc-editor.org/info/rfc7188>.

\subsection{Informative References}

[RFC5226] Narten, T. and H. Alvestrand, "Guidelines for Writing an IANA Considerations Section in RFCs", BCP 26, RFC 5226, DOI 10.17487/RFC5226, May 2008, <http://www.rfc-editor.org/info/rfc5226>. 
Acknowledgments

The authors would like to thank Adrian Farrel for pointing out the need to reorganize and rationalize the naming of the TLVs defined by [RFC5444] and Tom Taylor and the RFC Editor for pointing out some omissions and errors.

Authors' Addresses

Christopher Dearlove

BAE Systems Advanced Technology Centre

West Hanningfield Road

Great Baddow, Chelmsford

United Kingdom

Phone: +44 1245242194

Email: chris.dearlove@baesystems.com

URI: http://www.baesystems.com/

Thomas Heide Clausen

LIX, Ecole Polytechnique

Phone: +3366058 9349

Email: T.Clausendcomputer.org

URI: http://www.ThomasClausen.org/ 